\title{
BLOG@CACM
}

The Communications Web site, http://cacm.acm.org, features more than a dozen bloggers in the BLOG@CACM community. In each issue of Communications, we'll publish selected posts or excerpts.

Follow us on Twitter at http://twitter.com/blogCACM

\section{Can We Use Al for Global Good?}

\author{
Amir Banifatemi observes how the AIfor Good Summit \\ "allowed us to start a dialogue, find a common frame of reference, \\ and decide how our steps would be smart and structured."
}

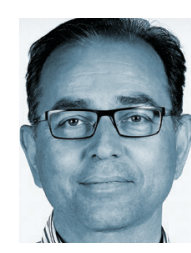

\section{Amir Banifatemi Validating Beneficial Al \\ https://cacm.acm. org/blogs/blog- \\ cacm/229283-validating-}

beneficial-ai/fulltext

July 3, 2018

Can the diverse artificial intelligence (AI) community come together to build an infrastructure to advance the United Nation's sustainable development goals (SDGs, https://sustainabledevelopment.un.org/sdgs) around the world? Can global projects be developed that begin to address pressing issues surrounding some of our greatest humanitarian challenges to help all?

Those were the goals of the second annual AI for Good Global Summit, the leading United Nations platform for dialogue on Artificial Intelligence held in Geneva, Switzerland, over three days in May.

The conference was organized by the International Telecommunication Union (ITU), the United Nations' specialized agency for information and communication technology (ICT), in partnership with the XPRIZE Foundation, the Association for Computing Machinery (ACM), and 32 sister UN agencies. The $500+$ attendees consisted of a diverse set of multistakeholders with wide-ranging expertise-from the individual UN agencies (including everything from UNESCO and UNICEF to The World Health Organization, The World Bank, and UNHCR), AI researchers, public- and private-sector decisionmakers, potential financial partners and sponsor organizations.

The focus of the 2018 edition of the AI for Good Summit was to bring together stakeholders prepared to propose practical projects to tackle topics within the 17 SDGs. Inspired by the XPRIZE incentive model, the goal was to present actual proposals in front of attendees to validate feasibility, timing, and how meaningful next steps can be identified. In short, setting actual solutions in motion.

As part of the summit design, AI innovators in attendance were connected with invited public- and private-sector decision-makers. Four breakthrough tracks-looking at satellite imagery, healthcare, smart cities, and trust in $\mathrm{AI}$ - set out to propose AI strategies with supporting projects to advance sustainable development. Teams were guided in this effort by an expert audience representing industry, academia, government, and civil society. Each track proposed projects, as well as introducing existing and future obstacles to the attendees, who then worked collaboratively to take promising strategies forward.

The results were demonstrative of a strong momentum and multistakeholder interest in collaboration to identify AI-based solutions with action at their core. The AI for Good Summit has achieved agreement on a community-oriented approach to support 35 projects, fast-tracked so they can be realized in as quickly as six months through a two- or three-year window. Priority projects coming out of each of the event tracks included:

- Developing Data and AI Commons: A transversal effort during the three days of the conference was 
designed to capture common core principles and opportunities to build a platform enabling beneficial AI. To provide AI to the masses, there is a need to have usable and shareable data in a common format that everyone can access. General datasets and relevant information useful to machine learning specialists is often spread throughout multiple repositories-there is an opportunity to consolidate them to level the playing field. This, for example, can be domain-specific, such as care, treatment, and outcomes for health researchers, historical weather data, satellite imagery, and landmass/ ocean temperature figures for agriculture and climate prediction, or city traffic, lighting, and crime statistics for city planners.

Data Commons would offer assemblies of datasets and supporting usage of AI tools, knowledge, and expertise of AI practitioners to launch new AI projects, scale up fast, and contribute new and improved resources to the AI for Good community. Data Commons would provide a foundation of the AI Commons, a global initiative proposed at the conclusion of the AI for Good Summit. AI Commons would help make access to AI capabilities universal and provide the public a platform to solve challenges with AI and drive inclusion.

The AI Commons is expected to be announced in late Q3 with opportunities for all stakeholders to join and participate in its development and deployment.

- AI-Powered Analysis of Satellite Imagery: Satellites transmit the equivalent of approximately two billion one-megapixel photographs every day, and AI is the only thing that can let us see the whole world at once. Beyond recording these images, they can create a global real-time database of the world. Three project proposals are focused on agriculture and use of AI-powered satellite imagery analysis to predict and prevent deforestation, pinpoint and track livestock, and provide data analytics to enable micro-insurance to smallholder family farming-small farms that rely mainly on family labor that are seen as the prime driver of agricultural production in developing countries.

\section{"We are seeing the Al community working together to create an infrastructure for responsible communication, development, and trust."}

An additional project proposal looks at creating a global service platformwith associated enabling infrastructure and common capabilities - that would allow developers to establish and support immediate scaling of new satellite data projects.

- AI and Healthcare: As one of the fastest-growing economic sectors in many countries, scalable technology surrounding the convergence of health and AI is exciting. Fifteen project proposals are moving forward, including predictive projects surrounding vision loss and osteoarthritis, integration and analysis of medical data, AI and healthcare policy, and responses to disease outbreak as well as other medical emergencies. There was also discussion surrounding the creation of a new, open study platform for stakeholders, supported by ITU and the World Health Organization, that would serve as a repository of use cases of AI in healthcare to identify data formats as well as interoperability mechanisms required to amplify their impact.

- Building Trust in AI: To build wellearned trust in the long term, Trustfactory.ai is being established as an incubator to research, source, support, and address key dimensions of trust in AI. The research collective is led by Cambridge University and the University of Padova-and stakeholders see this as a second leg of the infrastructure needed to expand AI usage globally.

- AI and Smart Cities: With the goal to identify common repositories of best practices, seven project proposals focus on the development of AI-driven simulations of city environments and bringing a humancentered approach to each vision. The projects support linguistic diversity within cities; the enabling of blockchain-based, citizen-centered decision making; strategies to combat gender imbalance and violence, and the use of AI to enhance the cultural heritage of each city to ensure that there are as many different definitions of a smart city as there are cities in the world. There is also a project to establish a global network-the 'Internet of Cities'-to share the data, knowledge, and expertise required to replicate successful smart cities around the world.

With collaborative efforts such as these, we are seeing the AI community working together to create an infrastructure for responsible communication, development, and trust. The foundational work that began at the first AI for Good Summit has allowed us to start a dialogue, find a common frame of reference, and decide how our steps would be smart and structured. Our focus this year was to accelerate progress, launching projects that will show tangible results and provide positive impact in key areas.

The cycle is set to continue. The 2019 summit will take stock of progress and will continue the focus on identifying practical ways to identify and implement AI for Good projects

This is a time of building infrastructure, guidelines, and kicking off focused development of tangible tools to accelerate the beneficial. Using AI for Good is the mantra that is gaining traction with more participation and conversations that make sense, and the conversation is not going to stop. AI innovations constitute one of the platforms that can bring benefits for everyone, and is a platform that can be a public asset for the common good. There is a great need to extend AI to more people and more places in a responsible way. We believe giving the public a common platform can benefit everyone.

Amir Banifatemi is the Group Lead, AI and Frontier Technologies for XPRIZE. 